\title{
ACTIVE TEMPERATURE COMPENSATION FOR MICROMACHINED RESONATORS
}

\author{
Matthew Hopcroft ${ }^{1}$, Renata Melamud ${ }^{1}$, Rob N. Candler ${ }^{1}$, Woo-Tae Park ${ }^{1}$, Bongsang Kim ${ }^{1}$, Gary Yama ${ }^{2}$, \\ Aaron Partridge ${ }^{2}$, Markus Lutz ${ }^{2}$, Thomas W. Kenny ${ }^{1}$ \\ ${ }^{1}$ Stanford University, \\ Departments of Mechanical and Electrical Engineering \\ Stanford, CA 94305 \\ ${ }^{2}$ Robert Bosch Corporation, \\ Research and Technology Center, North America \\ Palo Alto, CA 94304
}

\begin{abstract}
Encapsulated micromechanical resonators have been fabricated from single crystal silicon and a method for stabilizing their resonant frequency over temperature has been demonstrated. The method uses high-frequency AC signals applied directly to the resonant structure in order to heat it. By adjusting the power of the heating signal, varying amounts of heat could be added to the resonant structure in order to compensate for the effects of changing external temperature and the variation in resonant frequency was reduced significantly. This method requires minimal changes to existing resonator designs and is applicable to any resonator with a current path through its structure. The resonators examined here exhibited temperature coefficients of resonant frequency of over $210 \mathrm{~Hz} /{ }^{\circ} \mathrm{C}\left(170 \mathrm{ppm} /{ }^{\circ} \mathrm{C}\right)$ without temperature compensation. With compensation, the temperature coefficient was reduced to less than $5 \mathrm{~Hz} /{ }^{\circ} \mathrm{C}\left(4 \mathrm{ppm} /{ }^{\circ} \mathrm{C}\right)$.
\end{abstract}

\section{INTRODUCTION}

Micromachined resonators are of interest for applications in on-chip high frequency signal manipulation and integrated circuit timing [1, 2]. Silicon is a preferred material for highperformance micromechanical resonators because of its high mechanical stiffness and IC compatibility; however, it exhibits a temperature coefficient of stiffness as high as $75 \mathrm{ppm} /{ }^{\circ} \mathrm{C}$, and a significant temperature coefficient of expansion, $2.6 \mathrm{ppm} /{ }^{\circ} \mathrm{C}$. These effects cause resonators fabricated in silicon to exhibit large variations in resonant frequency over practical operating temperature ranges. Many important applications require a small variation of resonant frequency with temperature $\left(<1 \mathrm{ppm} /{ }^{\circ} \mathrm{C}\right)$, so there is interest in novel integrated temperature compensation approaches. This paper describes the first demonstration of resonator temperature compensation based on direct heating of the resonator structure within a wafer-scale encapsulation. This work is a first step towards our goal of a "micro-oven" consisting of a temperature-regulated encapsulation structure, and demonstrates the ability to merge the electrical connections necessary for thermal measurement, heating, and resonator operation with the wafer-scale hermetic encapsulation process previously developed $[3,4]$.

\section{FABRICATION}

The resonator designs chosen for these experiments are doubly-clamped tuning forks [5], shown in Figure 1. Each beam of the tuning fork measures $220 \mu \mathrm{m} \times 20 \mu \mathrm{m} \times 8 \mu \mathrm{m}$, and the device has a resonant frequency and quality factor $(\mathrm{Q})$ of approximately $1.24 \mathrm{MHz}$ and 12,000 respectively at $23^{\circ} \mathrm{C}$.

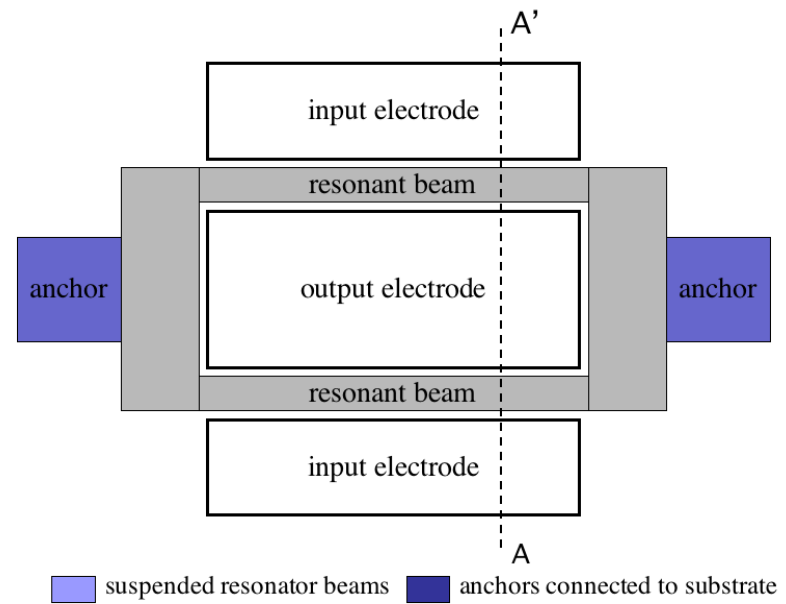

Figure 1 Schematic plan view of the double-clamped tuning fork resonator design.

The resonators are fabricated in a silicon-based, ICcompatible, encapsulated process [3, 4], illustrated in Figure 2. Structures are defined in the device layer of an SOI wafer and then encapsulated with a layer of epitaxial silicon. Openings are etched to allow HF vapor release of the resonator, and the final seal is completed with a second epitaxial silicon deposition. A SEM cross-section of a device, taken after the encapsulation has been deposited, is shown in Figure 3.

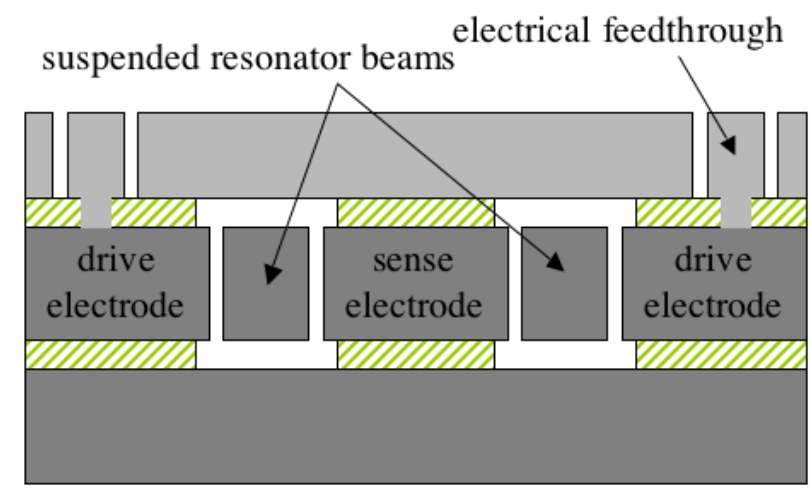

$\square$ Si $\square$ oxide $\square$ epitaxial Si

Figure 2 Schematic cross-section of the encapsulated silicon resonators. The section is from the line A-A' in Figure 1. Electrical feedthroughs permit contact with the encapsulated device. The substrate and device layers are single crystal silicon, and the top encapsulation layer is epitaxial polysilicon.

Travel support has been generously provided by the Transducers Research Foundation and by the DARPA MEMS and DARPA BioFlips programs. 


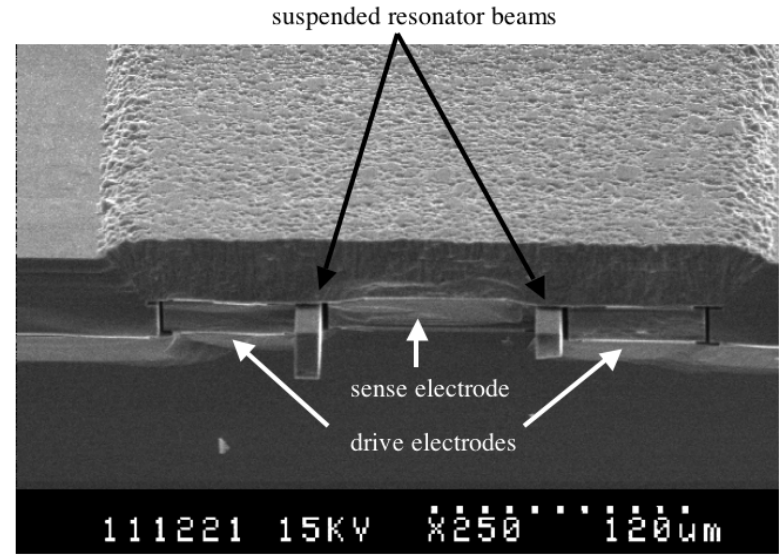

Figure 3 SEM cross-section of a resonator during fabrication. The section is approximately on the line A-A' in Figure 1, with the resonator beams protruding.

\section{THEORY}

Silicon is an attractive material for microresonator construction because it can be produced with a nearly ideal crystal structure, which should allow for perfectly reproducible. fatiguefree mechanical operation. However, like many semiconductors, silicon exhibits a high material sensitivity to temperature. Values found in literature for temperature coefficient of stiffness are as high as $75 \mathrm{ppm} /{ }^{\circ} \mathrm{C}$, and a temperature coefficient of expansion of $2.6 \mathrm{ppm} /{ }^{\circ} \mathrm{C}$. These effects cause the resonant frequency of silicon resonators to exhibit a strong temperature dependence [6]. Figure 4 shows the measured change in resonant frequency of a typical double-clamped tuning fork micromechanical resonator plotted against temperature. The change in resonant frequency with temperature is $214 \mathrm{~Hz} /{ }^{\circ} \mathrm{C}$, or $173 \mathrm{ppm} /{ }^{\circ} \mathrm{C}$. The relationship is linear in this temperature range. This large temperature coefficient of frequency is attributed to differential thermal expansion between the device layer and the encapsulation layer leading to temperature-induced stress in the resonator.

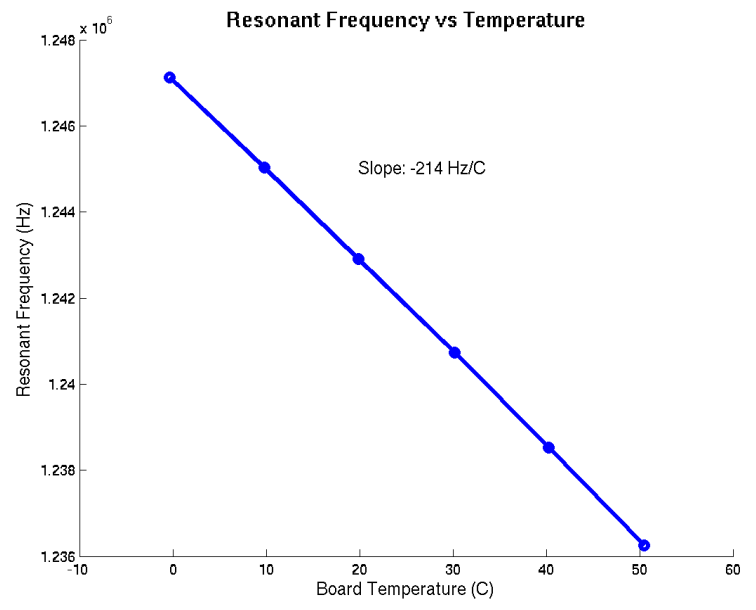

Figure 4 Plot of resonator resonant frequency $v$ s temperature for a double-clamped tuning fork resonator with a resonant frequency of $1.24 \mathrm{MHz}$ at $25^{\circ} \mathrm{C}$. This represents a sensitivity to temperature of $173 \mathrm{ppm} /{ }^{\circ} \mathrm{C}$.
Our approach to alleviating this dependence on external temperature is to add heat directly to the resonator beam. For these experiments, a high-frequency AC signal was applied to the beams to raise their temperature by Joule heating. The heating power could then be adjusted as necessary to compensate for the effects of external temperature. A $5 \mathrm{MHz}$ signal frequency was chosen for applying heat power so that the additional voltage on the resonator beams would not interfere with the resonant frequency measurement. Figure 5 shows the relationship between applied heating power and resulting resonant frequency, at various temperatures. The resulting plots are linear, as we expect from the temperature data in Figure 4.

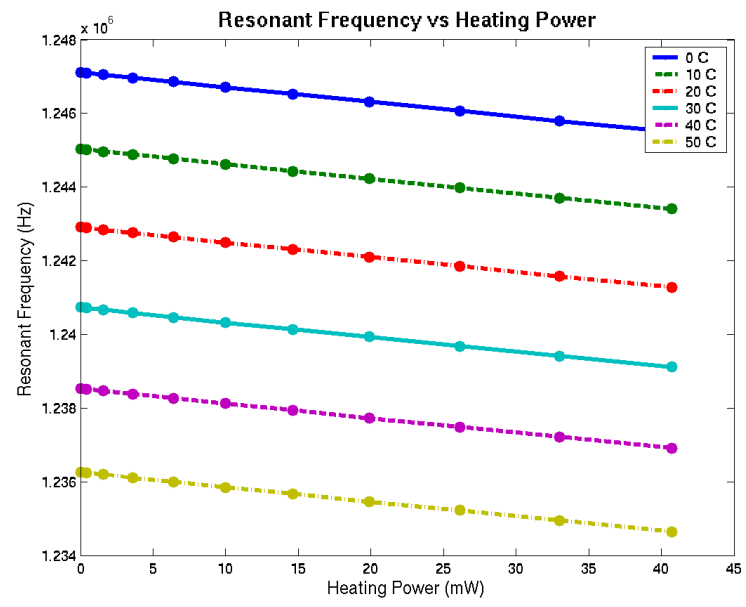

Figure 5 Plot of resonator resonant frequency vs applied heating power, at various temperatures. The maximum power applied was limited by the capability of the function generator used to generate the heating signal. This corresponds to a maximum frequency change of approximately $1700 \mathrm{ppm}$.

There are two ways in which the applied heating power can cause a shift in resonant frequency. First, there is heating of the resonator structure, which causes softening of the bulk modulus of the silicon and compressive stress in the resonator beams due to differential thermal expansion of the beam, the substrate, and the encapsulation layers. There are many unknowns related to heating the resonator structure, including the initial stress in the beam, compressive stress in the beam due to thermal expansion, additional stress induced by the packaging, and the temperature conditions inside the encapsulated cavity. It is clear that the temperature distribution in the resonator due to the applied heating power is not uniform, unlike the temperature distribution due to environmental temperature, and the specific interactions governing the relationship between resonant frequency and temperature are complex.

A finite element model of the microresonator is being developed to study the interactions between external/internal temperature, heater current, and resonant frequency. In this model, we begin by calculating the distribution of the electric current throughout the beam, and then extract the heat deposition as a function of location. A thermal model of the resonator takes the heat input information and computes the temperature distribution within the resonator. This temperature distribution can be used to calculate the local change in bulk modulus as well as thermal expansion effects on the dimensions and effective stiffness of the resonator, and so the resonant frequency can be calculated. Some modeling results for the temperature of the silicon beam are shown in Figure 6. At maximum heating power, the peak temperature of 
the beam is expected to be $9-12^{\circ} \mathrm{C}$ above the ambient, at room temperature $\left(23^{\circ} \mathrm{C}\right)$. This correlates with the measured change in resonant frequency, which corresponds to an approximate $10^{\circ} \mathrm{C}$ change in temperature.

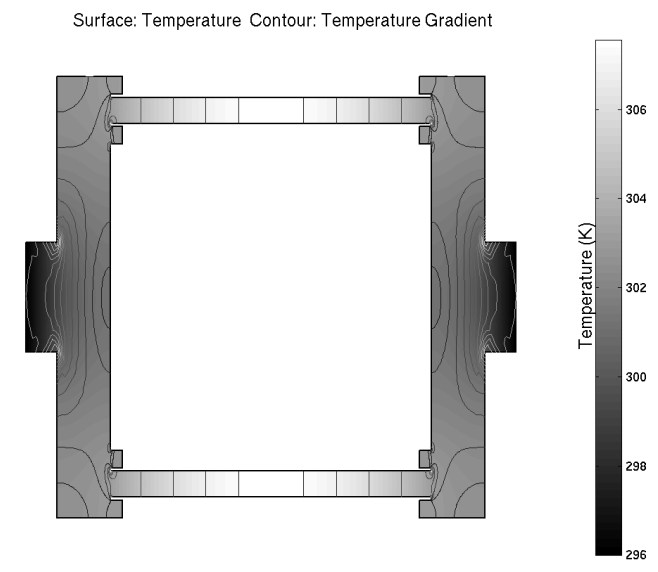

Figure 6 Plot of calculated temperature distribution due to Joule heating of the resonator beam. Higher temperatures are lighter colors. The temperature gradients are shown by the contour lines.

The second way in which the applied heating power can cause a shift in the resonant frequency is an electrostatic spring softening effect due to the rectification of the AC heating voltage signal by the quadratic force-voltage relationship in the electrostatic actuator. In our doubly-clamped resonators, differential thermal expansion is dominant. We estimated that the change in resonant frequency due to spring softening was less than $80 \mathrm{ppm}$, which is the noise due to typical uncontrolled temperature drift. However, the response of the resonator to applied heating is measured, as in Figure 5, and this data is used to calibrate the compensation feedback process, so all of the contributions to resonant frequency change are included in the frequency compensation control.

\section{RESULTS}

The resonators were packaged in surface mount IC packages and mounted on printed circuit boards for testing. The temperature of the resonator environment was controlled using a Thermotron S-1.2 temperature chamber, which is capable of maintaining the air temperature in the chamber at $+/-0.1^{\circ} \mathrm{C}$. A semiconductor temperature sensor, the LM35 from National Instruments, was used to measure the temperature of the immediate resonator environment with $0.01{ }^{\circ} \mathrm{C}$ precision.

The resonators are actuated and measured electrostatically, and a DC electrostatic bias is applied to the resonator beams to increase the charge present in the capacitors. As discussed above, a $5 \mathrm{MHz}$ AC signal, generated by a SRS DS345 function generator, was applied to the resonator beams in order to compensate for the effects of external temperature. A circuit, based on the "Bias-T" of Clark, et al [7], was used to apply a DC electrostatic bias to the beam without affecting the DC bias required for the capacitive readout, as shown in Figure 7. The modified bias-T used here is basically high-pass and low-pass filters joined to allow the coupling of the DC bias and the heating signal. A DC blocking capacitor prevents the bias from creating a potential drop across the beam.

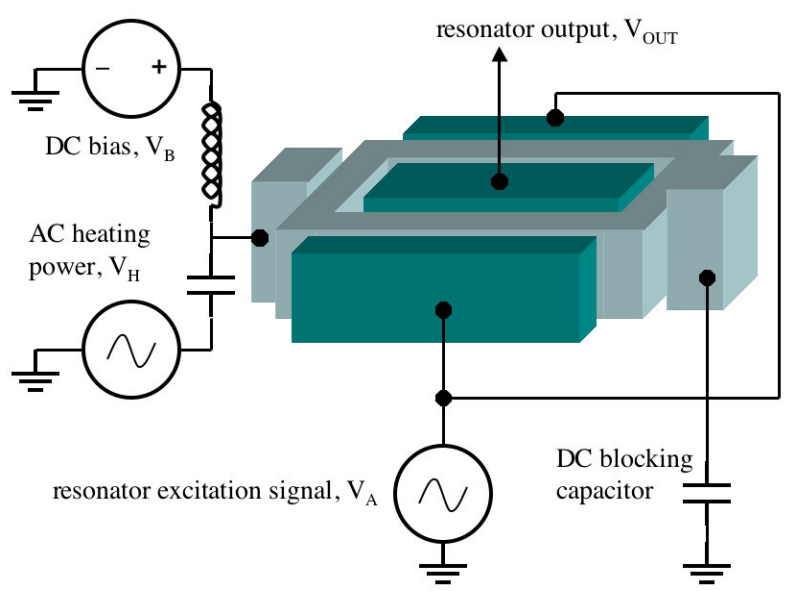

Figure 7 Electrical connections for measuring the resonator. A "modified Bias-T" circuit allows the AC heating signal to be applied to the resonator beam without allowing the DC bias voltage to create a potential drop across the beam.

The resonators were measured using a HP4395A network analyzer, which provided a sinusoidal actuation signal to the drive electrodes, and simultaneously measured the response at the sense electrode. The frequency of the actuation signal and input measurement are swept over a frequency range and the amplitude and phase of the response is recorded (divided by the actuation signal to cancel out the effects of any irregularities in the output). A typical resonator measurement is shown in Figure 8. The measurement system was automated so that measurements could be taken over long periods of time. The network analyzer built-in peak detection algorithm was used to determine the frequency at the maximum response, and hence the resonant frequency.
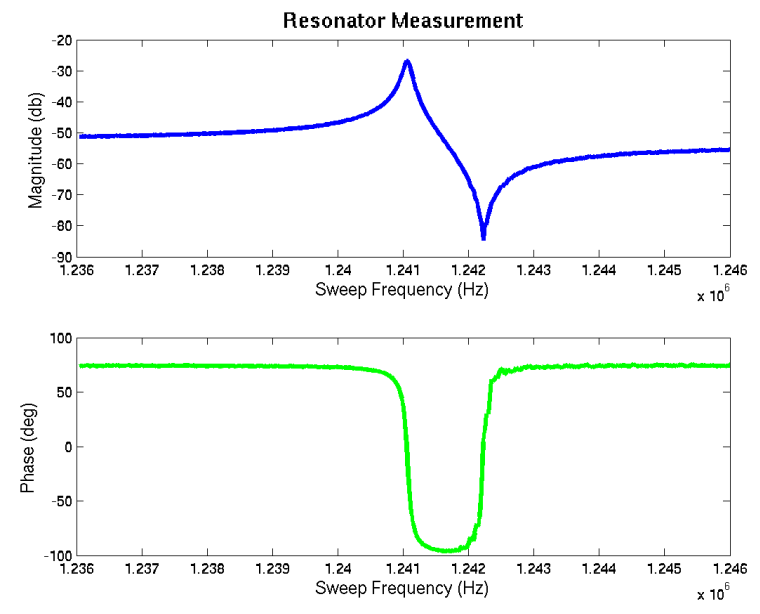

Figure 8 Bode plot of typical 1.24 MHz resonator response.

For temperature compensated operation, the temperature inside the chamber was measured. The output power that was required to keep the resonator temperature constant was then computed, based on the results of measurements of the responsiveness of the device to external temperature and to applied heating power (e.g. the data in Figures 4 and 5). This heating power was then applied to the resonator, and measurements were taken. Figure 9 compares the resonant frequency with and without temperature compensation as the ambient temperature is swept over $22-15^{\circ} \mathrm{C}$. The variation in frequency is reduced over $40 \mathrm{x}$, to 
$4 \mathrm{ppm} /{ }^{\circ} \mathrm{C}$, with temperature compensation. The relative temperature range for compensation experiments was limited to slightly less than $10^{\circ} \mathrm{C}$, as in Figures 5 and 9, by the amplitude output of the function generator.

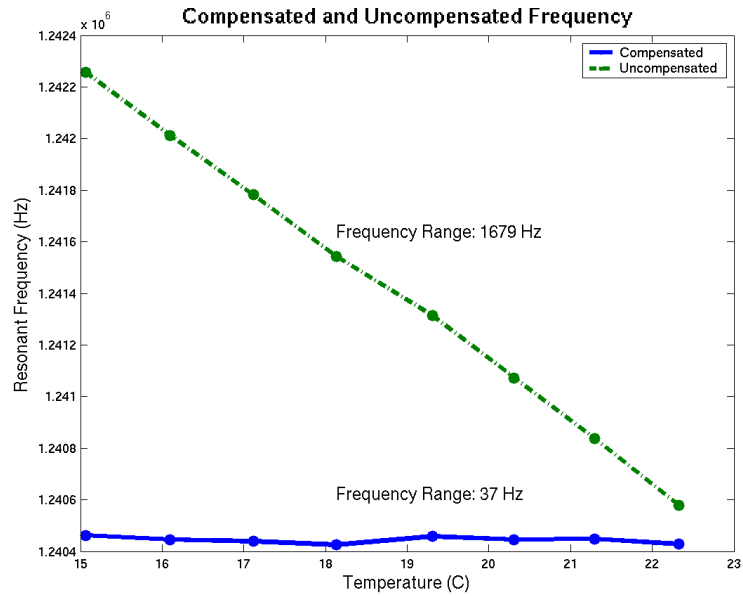

Figure 9 Plot of resonant frequency vs temperature. Each data point represents an average of 5 measurements, with an average deviation of $2.2 \mathrm{~Hz}$. The change in frequency without compensation is $170 \mathrm{ppm} /{ }^{\circ} \mathrm{C}$, and with compensation it is 4 $\mathrm{ppm} /{ }^{\circ} \mathrm{C}$.

Measuring the frequency of the resonator, as described above, required between 60 and 180 seconds to accomplish per measurement, depending on the resolution of the measurement. Temperature fluctuations during that time are the primary source of error in the measurements. These fluctuations were typically less than $0.05^{\circ} \mathrm{C}$, which corresponds to approximately $10 \mathrm{~Hz}(8$ $\mathrm{ppm}$ ) error in each individual measurement of resonant frequency. Averages of multiple measurements were used to reduce the experimental error to less than $5 \mathrm{~Hz}$.

\section{CONCLUSION}

A method for directly heating silicon micromechanical resonators in order to achieve resonant frequency stability over temperature has been demonstrated. This approach has the advantage of requiring limited modifications of existing designs, and general applicability to many other designs, and these devices represent an excellent platform for development of compensation approaches that can eventually lead to silicon resonators for highstability applications. There are some drawbacks in the requirements for external circuitry and the high-frequency signal source; more significantly, because the distribution of heating is uneven, the direct heating approach leads to significant temperature gradients throughout the resonators, and to complex effects on the resonant frequency of the structure. Nonetheless, the results presented here show the feasibility of the direct heating method.

The definitive solution to temperature stability issues is to situate the resonator inside an isothermal chamber with temperature regulation. Our ultimate goal is to produce such a device within a wafer-level encapsulation process. An intermediate step, which has been fabricated but not yet tested, involves localization of the heating at the edges of the resonator, so that the beams are uniformly heated, and more likely to follow a predictable thermal control scheme. Results from these devices are being gathered, and will be presented at the meeting. Future work will be focused on fabrication processes and operational methods for resonators that are encapsulated within truly thermallyregulated enclosures.

\section{ACKNOWLEDGEMENTS}

This work was supported by DARPA HERMIT (ONR N66001-03-1-8942), the Robert Bosch Corporation Palo Alto Research and Technology Center, a CIS Seed Grant, The National Nanofabrication Users Network facilities funded by the National Science Foundation under award ECS-9731294, and The National Science Foundation Instrumentation for Materials Research Program (DMR 9504099).

The authors would also like to acknowledge the assistance of Klint A. Rose of Stanford University with some problems in computational methods.

\section{REFERENCES}

Nguyen, C.T. Micromechanical components for miniaturized low-power communications (invited plenary). in IEEE MTT-S International Microwave Symposium RF MEMS Workshop on Microelectromechanical Devices for RF Systems: Their Construction, Reliability, and Application. 1999.

Anaheim, California,, pp. 48-77.: IEEE. Nguyen, C.T. Transceiver Front-End Architectures Using Vibrating Micromechanical Signal Processors. in Topical Meeting on Silicon Monolithic Integrated Circuits in RF Systems. 2001. Ann Arbor, Michigan, USA: IEEE.

Partridge, A., et al. New thin film epitaxial polysilicon encapsulation for piezoresistive accelerometers. in Micro Electro Mechanical Systems, 2001. The 14th IEEE International Conference on. 2001.

Park, W.-T., et al. Wafer Scale Encapsulation of MEMS Devices. in InterPack 2003, International Packaging and Technical Conference and Exhibition. 2003. Maui, Hawaii USA.

Candler, R.N., et al. Investigation of Energy Loss Mechanisms in Micromechanical Resonators. in Transducers '03. 2003. Boston, MA USA.

Hsu, W.-T. and C.T.-C. Nguyen. Geometric stress compensation for enhanced thermal stability in micromechanical resonators. in Ultrasonics Symposium, 1998. Proceedings., IEEE. 1998.

Clark, J.R. and C.T.-C. Nguyen. Measurement techniques for capacitively-transduced VHF-to-UHF micromechanical resonators. in Transducers '01 I Eurosensors $X V$, The 11th International Conference on Solid-State Sensors and Actuators. 2001. Munich, Germany: Springer. 